\title{
Cultivar and Plant Arrangement Effects on Yield and Fruit Quality of Bell Pepper
}

\author{
Brian A. Kahn ${ }^{1,2}$ \\ Department of Horticulture and Landscape Architecture, 360 Agricultural \\ Hall, Oklahoma State University, Stillwater, OK 74078-6027
}

Daniel I. Leskovar ${ }^{1}$

Texas Agricultural Experiment Station, Vegetable and Fruit Improvement Center, Texas A\&M University, 1619 Garner Field Road, Uvalde, TX 78801-6205

\section{Additional index words. Capsicum annuum, crop geometry, spacing}

\begin{abstract}
Single- and double-row arrangements of a fixed population (one plant every $0.285 \mathrm{~m}^{2}$ ) were compared in factorial combination with two (2002) or five (2003) cultivars for effects on yield and fruit quality of bell pepper (Capsicum annuum L.). Arrangements for 2002 were S30, single rows $0.95 \mathrm{~m}$ apart, plants within rows $30 \mathrm{~cm}$ apart; D30, $1.9 \mathrm{~m}$ between centers of double-row beds, double rows $30 \mathrm{~cm}$ apart on beds, plants within rows $30 \mathrm{~cm}$ apart; $\mathrm{S37.5}$, single rows $0.76 \mathrm{~m}$ apart, plants within rows $37.5 \mathrm{~cm}$ apart; and D37.5, $1.52 \mathrm{~m}$ between centers of double-row beds, double rows $24 \mathrm{~cm}$ apart on beds, plants within rows $37.5 \mathrm{~cm}$ apart. Only the S30 and D30 arrangements were used in 2003 after 2002 results showed almost no differences between S30 and S37.5 or between D30 and D37.5. Choice of cultivar was more critical in Texas, where ' $X 3 R$ Wizard' consistently outperformed 'King Arthur', than in Oklahoma. Single rows resulted in more full-season total marketable fruit weight than double rows in three experiments out of four, primarily as a result of an increased weight of U.S. No. 1 fruit with single rows. Average weight per marketable fruit was consistently unaffected by plant arrangement. Single rows also resulted in a greater full-season weight of sunburned fruit than double rows in two experiments out of four. Cultivar $\times$ plant arrangement interactions were not evident in Oklahoma and never involved full-season marketable fruit weights at either location in either year. Given the tested population, a single-row arrangement is likely to result in increased full-season production of U.S. No. 1 bell pepper fruit compared with a double-row arrangement, despite an increased potential for sunburned fruit with single rows.
\end{abstract}

In a competitive marketplace, total yield may be less important than yield of a highquality, premium product. Selection of adapted cultivars and appropriate cultural practices are important steps toward this goal.

Many studies have been published on plant populations for bell peppers (Ahmed, 1984; Batal and Smittle, 1981; Gaye et al., 1992; Locascio and Stall, 1994; Stoffella and

\footnotetext{
Received for publication 8 May 2006. Accepted for publication 30 July 2006.

The Oklahoma portion of this research was supported in part under project H-2026. Appreciation is extended to the U.S. Department of Agriculture for a grant (CSREES 2003-34402-13647 "Designing Foods for Health") for partial funding in Texas. Approved for publication by the director, Oklahoma Agricultural Exp. Sta. The information given in this publication is for educational purposes only. Mention of a trademark, proprietary product, or vendor does not constitute a guarantee or warranty of the product, nor does it imply approval or disapproval to the exclusion of other products or vendors that may also be suitable.

${ }^{1}$ Professor.

${ }^{2}$ To whom reprint requests should be addressed; e-mail brian.kahn@okstate.edu
}

shading the fruit better than in the single rows.

The current study was designed to determine whether different arrangements of a given plant population would affect yield of premium (U.S. Fancy and U.S. No. 1) bell pepper fruit. The comparison of single- and double-row arrangements also tested the hypothesis that use of double rows might reduce the number of cull fruits. Yield and grade-out of different cultivars were also examined, and possible cultivar $\times$ plant arrangement interactions were tested.

\section{Materials and Methods}

Studies were conducted at the Oklahoma Vegetable Research Station, Bixby, and at the Texas Agricultural Experiment Station, Uvalde, in 2002 and 2003. The soil at Bixby was a Severn very fine sandy loam [coarsesilty, mixed (calcareous), thermic Typic Udifluvent]. The soil at Uvalde was a Uvalde silty clay loam (fine-silty, mixed, hyperthermic Aridic Calciustoll). No plastic mulches were used at either location. Weeds were controlled with herbicides supplemented by hand and machine cultivation. Plant water requirements were met with rainfall supplemented by overhead sprinkler irrigation at Bixby and by surface drip irrigation at Uvalde. Standard foliar insecticides, fungicides, and bactericides were applied as needed.

The bell pepper cultivars King Arthur and X3R Wizard were used both years. Additional cultivars in 2003 were Boynton Bell, Karma, and Lafayette. Transplants were commercially grown in 2002 and grown onsite in 2003. In both cases, transplants were produced in flats with inverted pyramid cells (200 cells per flat; volume, $18 \mathrm{~cm}^{3}$ per cell).

Single- and double-row arrangements of a fixed population (one plant every $0.285 \mathrm{~m}^{2}$ ) were compared. This population was chosen as a rough average of recommended plant population densities for Oklahoma and Texas. Arrangements for 2002 were S30, single rows $0.95 \mathrm{~m}$ apart, plants within rows $30 \mathrm{~cm}$ apart; D30, $1.9 \mathrm{~m}$ between centers of double-row beds, double rows $30 \mathrm{~cm}$ apart on beds, plants within rows $30 \mathrm{~cm}$ apart; S37.5, single rows $0.76 \mathrm{~m}$ apart, plants within rows $37.5 \mathrm{~cm}$ apart; and D37.5, $1.52 \mathrm{~m}$ between centers of double-row beds, double rows 24 $\mathrm{cm}$ apart on beds, plants within rows $37.5 \mathrm{~cm}$ apart. Only the S30 and D30 arrangements were used in 2003 after 2002 results showed almost no differences between S30 and S37.5 or between D30 and D37.5.

The experimental design was a split plot. In 2002, arrangements were main plots in a $4 \times 4$ Latin square; cultivars were subplots. In 2003 , cultivars were main plots in a randomized complete block with four replications; arrangements were subplots.

Plot lengths varied by plant arrangement. In 2002, single-row treatments had 32 plants per row (16 per cultivar). Double-row treatments had 20 plants per row (10 per cultivar). In 2003, S30 had 16 plants per row and D30 
had 10 plants per row. In all cases, areas containing 12 plants per plot were designated for data collection, and appropriately spaced guard rows separated the main plots.

Selective hand harvests of mature green fruits were made periodically. Fruits were classified as U.S. Fancy, U.S. No. 1, U.S. No. 2, and culls, according to U.S. Dept. of Agriculture grading standards. Cull fruits were further classified by predominant reason for culling as follows: blossom-end rot, sunburn, or other defects (such as seriously misshapen or insect damaged)

\section{Cultural Practices}

Bixby, 2002. The soil was prepared with a broadcast preplant-incorporated application of $31 \mathrm{~N}-14 \mathrm{P}-26 \mathrm{~K} \mathrm{~kg} \cdot \mathrm{ha}^{-1}$, plus trifluralin [2,6-dinitro- $N, N$-dipropyl-4-(trifluoromethyl) benzenamine at $560 \mathrm{~g} \cdot \mathrm{ha}^{-1}$ ] for weed control. Transplants were set in the field by hand on 17 Apr. Each plant received $\approx 240$ $\mathrm{mL}$ starter solution providing $1079 \mathrm{~N}-941 \mathrm{P}$ $895 \mathrm{~K} \mathrm{mg} \cdot \mathrm{L}^{-1}$, plus diazinon ([O,O-diethyl $O$-[6-methyl-2-(1-methylethyl)-4-pyrimidinyl]phosphorothioate at $\left.300 \mathrm{mg} \cdot \mathrm{L}^{-1}\right)$. Plants were top dressed with urea to supply $56 \mathrm{~kg} \cdot \mathrm{ha}^{-1}$ $\mathrm{N}$ per application on 15 May and 4 June.

A total of 10 harvests were made beginning on 3 July and ending on 26 Aug. "Early harvest" was defined as the first four picks. On 27 Aug., three plants per plot were measured to the nearest centimeter for height from the soil to the highest growing point. Each plant then was cut at soil level, defruited, dried for $\approx 7 \mathrm{~d}$ at $55{ }^{\circ} \mathrm{C}$, and weighed.

Uvalde, 2002. The soil was prepared with a broadcast preplant-incorporated application of $70 \mathrm{~N}-50 \mathrm{P}-0 \mathrm{~K} \mathrm{~kg} \cdot \mathrm{ha}^{-1}$. The herbicide S-metolachlor (2-chloro- $N$-(2-ethyl-6-methylphenyl)- $N$-[(1S)-2-methoxy-1-methylethy-
1] acetamide at $1.28 \mathrm{~kg} \cdot \mathrm{ha}^{-1}$ ) was applied on 29 Mar., followed by $13 \mathrm{~mm}$ sprinkler irrigation for incorporation. Transplants were set in the field by hand on 10 Apr. Fertilizer (ammonium nitrate, urea, and phosphoric acid sources) was applied through the drip system on 18 Apr., 3 May, and 11 June to supply a supplemental total of $40 \mathrm{~N}-10 \mathrm{P}$ $\mathrm{kg} \cdot \mathrm{ha}^{-1}$.

A total of six harvests were made beginning on 19 June and ending on 28 Aug. "Early harvest" was defined as the first two picks. On 28 Aug., four plants per plot were measured for plant height, cut, and defruited as in Oklahoma. Sampled plants were dried for $6 \mathrm{~d}$ at $65^{\circ} \mathrm{C}$, and weighed.

Bixby, 2003. The soil was prepared with a broadcast preplant-incorporated application of trifluralin $560 \mathrm{~g} \cdot \mathrm{ha}^{-1}$. Transplants were set in the field by hand on $22 \mathrm{Apr}$. Each plant received starter solution as in 2002. Preplant $\mathrm{N}$ had not been applied, so a top dressing with urea to supply $56 \mathrm{~kg} \cdot \mathrm{ha}^{-1} \mathrm{~N}$ was made on 29 Apr. Adequate $\mathrm{P}$ and $\mathrm{K}$ were available from fertilization of previous trials. Plants also were top dressed with urea to supply $56 \mathrm{~kg} \cdot \mathrm{ha}^{-1} \mathrm{~N}$ per application on 21 May and 19 June, and to supply $28 \mathrm{~kg} \cdot \mathrm{ha}^{-1} \mathrm{~N}$ on $21 \mathrm{July}$.

A total of five harvests were made beginning on 24 June and ending on 28 July. "Early harvest" was defined as the first two picks. On 1 Aug., three plants per plot were measured for height and cut for dry weight determination as in 2002.

Uvalde, 2003. The soil was prepared with a broadcast preplant-incorporated application of $70 \mathrm{~N}-50 \mathrm{P}-0 \mathrm{~K}-2 \mathrm{Zn} \mathrm{kg} \cdot \mathrm{ha}^{-1}$. Transplants were set in the field by hand on $3 \mathrm{Apr}$. The herbicide S-metolachlor $\left(1.28 \mathrm{~kg} \cdot \mathrm{ha}^{-1}\right)$ was applied on 4 Apr. Fertilizer (ammonium nitrate and phosphoric acid sources) was applied through the drip system on 22 Apr. 2 May, and 2 June to supply a supplemental total of $45 \mathrm{~N}-30 \mathrm{P} \mathrm{kg} \cdot \mathrm{ha}^{-1}$. A total of nine harvests were made beginning on 17 June and ending on 18 Sept. "Early harvest" was defined as the first four picks. On 4 Aug., four plants per plot were measured for height and cut for dry weight determination as in 2002

\section{Results}

Early harvest, 2002. 'King Arthur' had more early fruit production than 'X3R Wizard' in Oklahoma, but not in Texas (Table 1). Single rows resulted in a greater overall total early fruit weight than double rows in Oklahoma, but not in Texas, and this was attributed to a greater weight of U.S. No. 1 fruit from single rows in Oklahoma (Table 1). No differences were found between the S30 and S37.5 arrangements, nor between the D30 and D37.5 arrangements. Only one interaction was evident, affecting early cull fruit weight in Texas. Both single-row arrangements of 'King Arthur' led to higher early cull weights than the D30 arrangement of 'King Arthur' and 'X3R Wizard' in Texas. The S30 arrangement of 'King Arthur' also led to a higher early cull weight than the S37.5 arrangement of 'X3R Wizard' (interaction means not presented).

Full-season yields, Oklahoma, 2002. 'King Arthur' produced higher marketable and overall total weights of fruit than 'X3R Wizard', and the average weight per marketable fruit also was greater for 'King Arthur' than for 'X3R Wizard' (Table 2). Plant arrangement effects were evident only for cull fruit production. Single rows led to higher cull weights than double rows. Effects were seen for both sunburned fruit and fruit with blossom-end rot (Table 2). No differences were found between the S30 and S37.5 arrangements, nor between the D30 and

Table 1. Bell pepper fruit yields from early harvest as affected by cultivar and plant arrangement, Bixby, Okla. (OK) and Uvalde, Texas (TX), 2002. ${ }^{2}$

\begin{tabular}{|c|c|c|c|c|c|c|c|c|c|c|c|c|c|c|c|}
\hline \multirow[b]{3}{*}{ Treatment factor } & \multicolumn{11}{|c|}{ Marketable fruit wt } & & & & \\
\hline & \multirow{2}{*}{$\begin{array}{l}\text { U.S. Fancy } \\
\frac{\left({\left.\mathrm{Mg} \cdot \mathrm{ha}^{-1}\right)}^{\mathrm{OK}^{v}}\right.}{}\end{array}$} & \multicolumn{2}{|c|}{$\begin{array}{l}\text { U.S. No. 1 } \\
\left(\mathrm{Mg} \cdot \mathrm{ha}^{-1}\right)\end{array}$} & \multicolumn{2}{|c|}{$\begin{array}{l}\text { Premium } \\
\left(\mathrm{Mg}^{\mathrm{x}} \cdot \mathrm{ha}^{-1}\right)\end{array}$} & \multicolumn{2}{|c|}{$\begin{array}{l}\text { U.S. No. 2 } \\
\left(\mathrm{Mg} \cdot \mathrm{ha}^{-1}\right)\end{array}$} & \multicolumn{2}{|c|}{$\begin{array}{c}\text { Total }^{\mathrm{w}} \\
\left(\mathrm{Mg} \cdot \mathrm{ha}^{-1}\right)\end{array}$} & \multicolumn{2}{|c|}{$\begin{array}{l}\text { Average } \\
\text { (g/fruit) }\end{array}$} & \multicolumn{2}{|c|}{$\begin{array}{l}\text { Cull fruit wt } \\
\left(\mathrm{Mg} \cdot \mathrm{ha}^{-1}\right)\end{array}$} & \multicolumn{2}{|c|}{$\begin{array}{l}\text { Overall totaly } \\
\text { fruit wt }\left(\mathrm{Mg} \cdot \mathrm{ha}^{-1}\right)\end{array}$} \\
\hline & & OK & TX & $\overline{\mathrm{OK}}$ & TX & OK & TX & $\overline{\mathrm{OK}}$ & TX & $\overline{\mathrm{OK}}$ & TX & OK & $\mathrm{TX}^{\mathrm{u}}$ & $\mathrm{OK}$ & TX \\
\hline King Arthur & 0.7 & 6.4 & 2.9 & 7.1 & 2.9 & 0.9 & 1.0 & 8.0 & 3.9 & 156 & 99 & 1.0 & 1.4 & 8.9 & 5.3 \\
\hline X3R Wizard & 0.1 & 2.1 & 3.5 & 2.2 & 3.5 & 0.3 & 1.2 & 2.5 & 4.7 & 140 & 106 & 0.3 & 1.2 & 2.9 & 5.9 \\
\hline Significance & $* *$ & ** & NS & $* *$ & NS & NS & NS & $* *$ & NS & $* *$ & NS & ** & NS & ** & NS \\
\hline \multicolumn{16}{|l|}{ Plant arrangement ${ }^{t}$} \\
\hline S37.5 & 0.4 & 4.4 & 3.2 & 4.8 & 3.2 & 0.7 & 1.3 & 5.5 & 4.5 & 142 & 106 & 0.5 & 1.4 & 6.1 & 5.9 \\
\hline D37.5 & 0.4 & 3.8 & 3.6 & 4.2 & 3.6 & 0.5 & 1.0 & 4.6 & 4.6 & 152 & 103 & 0.5 & 1.3 & 5.1 & 6.0 \\
\hline \multicolumn{16}{|c|}{ Contrasts within plant arrangements } \\
\hline Single vs. double rows & NS & * & NS & NS & NS & NS & NS & NS & NS & NS & NS & NS & NS & * & NS \\
\hline
\end{tabular}

${ }^{2}$ In Oklahoma, early harvest occurred on 3, 8, 12, and 17 July. None of the measured variables showed a significant $(P \leq 0.05)$ cultivar $\times$ plant arrangement interaction. In Texas, early harvest occurred on 19 June and 8 July. Only cull fruit weight showed a significant $(P=0.028)$ cultivar $\times$ plant arrangement interaction. ${ }^{y}$ Overall total $=$ U.S. Fancy + U.S. No. $1+$ U.S. No. $2+$ all culls.

${ }^{x}$ Premium $=$ U.S. Fancy + U.S. No. 1.

${ }^{\mathrm{w}}$ Total marketable = U.S. Fancy + U.S. No. $1+$ U.S. No. 2.

"There were no U.S. Fancy fruit at early harvest in Texas.

uSignificant $(P=0.05)$ cultivar $\times$ plant arrangement interaction; see text for details.

${ }^{\mathrm{t}} \mathrm{S} 30=$ single rows $0.95 \mathrm{~m}$ apart, plants $30 \mathrm{~cm}$ apart within rows; $\mathrm{D} 30=$ double rows $30 \mathrm{~cm}$ apart on beds, $1.9 \mathrm{~m}$ between centers of beds, plants $30 \mathrm{~cm}$ apart within rows; $\mathrm{S} 37.5$ = single rows $0.76 \mathrm{~m}$ apart, plants $37.5 \mathrm{~cm}$ apart within rows; D37.5 = double rows $24 \mathrm{~cm}$ apart on beds, $1.52 \mathrm{~m}$ between centers of beds, plants $37.5 \mathrm{~cm}$ apart within rows.

${ }^{\mathrm{s}}$ Contrasts for S30 vs. S37.5 and for D30 vs. D37.5 were ns for all variables in this table.

NS, *,** Not significant or significant by $F$ test at $P \leq 0.05$ or $\leq 0.01$ respectively. 
Table 2. Full-season bell pepper fruit yields as affected by cultivar and plant arrangement, Bixby, Okla., 2002. ${ }^{\mathrm{z}}$

\begin{tabular}{|c|c|c|c|c|c|c|c|c|c|c|}
\hline \multirow[b]{2}{*}{ Treatment factor } & \multicolumn{6}{|c|}{ Marketable fruit wt } & \multicolumn{3}{|c|}{ Cull fruit wt $\left(\mathrm{Mg} \cdot \mathrm{ha}^{-1}\right)$} & \multirow{2}{*}{$\begin{array}{c}\text { Overall totaly } \\
\text { fruit wt } \\
\left(\mathrm{Mg} \cdot \mathrm{ha}^{-1}\right)\end{array}$} \\
\hline & $\begin{array}{l}\text { U.S. Fancy } \\
\left(\mathrm{Mg} \cdot \mathrm{ha}^{-1}\right)\end{array}$ & $\begin{array}{l}\text { U.S. No. } 1 \\
\left(\mathrm{Mg} \cdot \mathrm{ha}^{-1}\right)\end{array}$ & $\begin{array}{l}\text { Premium }^{\mathrm{x}} \\
\left(\mathrm{Mg} \cdot \mathrm{ha}^{-1}\right)\end{array}$ & $\begin{array}{l}\text { U.S. No. } 2 \\
\left(\mathrm{Mg} \cdot \mathrm{ha}^{-1}\right)\end{array}$ & $\begin{array}{c}\text { Total }^{\mathrm{w}} \\
\left(\mathrm{Mg} \cdot \mathrm{ha}^{-1}\right)\end{array}$ & $\begin{array}{l}\text { Average } \\
\text { (g/fruit) }\end{array}$ & $\begin{array}{c}\begin{array}{c}\text { Sunburn } \\
\text { only }\end{array} \\
\text { ent }\end{array}$ & $\begin{array}{l}\text { Blossom end } \\
\text { rot only }\end{array}$ & $\begin{array}{l}\text { Total of } \\
\text { all culls }\end{array}$ & \\
\hline \multicolumn{11}{|l|}{ Cultivar } \\
\hline Significance & $* *$ & $* *$ & $* *$ & $* *$ & $* *$ & $* *$ & $* *$ & NS & $* *$ & $* *$ \\
\hline \multicolumn{11}{|l|}{ Plant arrangement ${ }^{\mathrm{u}}$} \\
\hline $\mathrm{S} 30$ & 0.4 & 23.0 & 23.4 & 10.9 & 34.4 & 105 & 2.1 & 3.0 & 9.0 & 43.3 \\
\hline D37.5 & 0.9 & 25.8 & 26.7 & 10.0 & 36.7 & 108 & 1.4 & 0.8 & 5.5 & 42.2 \\
\hline \multicolumn{11}{|c|}{ Contrasts within plant arrangements ${ }^{\mathrm{t}}$} \\
\hline Single vs. double rows & NS & NS & NS & NS & NS & NS & * & $* *$ & ** & NS \\
\hline
\end{tabular}

${ }^{z}$ Totals over 10 harvests from 3 July through 26 Aug. None of the measured variables showed a significant $(P \leq 0.05)$ cultivar $\times$ plant arrangement interaction.

${ }^{y}$ Overall total $=$ U.S. Fancy + U.S. No. $1+$ U.S. No. $2+$ all culls.

${ }^{x}$ Premium $=$ U.S. Fancy + U.S. No. 1.

${ }^{\mathrm{w}}$ Total marketable $=$ U.S. Fancy + U.S. No. $1+$ U.S. No. 2.

${ }^{\mathrm{v}}$ All culls includes misshapen fruit and fruit affected by sunburn, blossom-end rot, or plant pathogens.

${ }^{\mathrm{S}} \mathrm{S} 30=$ single rows $0.95 \mathrm{~m}$ apart, plants $30 \mathrm{~cm}$ apart within rows; D30 = double rows $30 \mathrm{~cm}$ apart on beds, $1.9 \mathrm{~m}$ between centers of beds, plants $30 \mathrm{~cm}$ apart within rows; S37.5 = single rows $0.76 \mathrm{~m}$ apart, plants $37.5 \mathrm{~cm}$ apart within rows; D37.5 = double rows $24 \mathrm{~cm}$ apart on beds, $1.52 \mathrm{~m}$ between centers of beds, plants 37.5 $\mathrm{cm}$ apart within rows.

${ }^{\mathrm{t}}$ Contrasts for S30 vs. S37.5 and for D30 vs. D37.5 were ns for all variables in this table.

Ns, *, ** Not significant or significant by $F$ test at $P \leq 0.05$ or $\leq 0.01$ respectively.

D37.5 arrangements. No cultivar $\times$ plant arrangement interactions were evident.

Full-season yields, Texas, 2002. 'X3R Wizard' produced a higher total weight of fruit than 'King Arthur', but the average weight per marketable fruit did not differ between the two cultivars (Table 3 ). This was a contrast to cultivar performance in Oklahoma (Table 2). Single rows resulted in a greater total marketable fruit weight than double rows, with higher weights from single rows in all marketable fruit categories except U.S. Fancy (Table 3). Plant arrangement effects on cull fruit production were less clear than in Oklahoma, because cultivar $\times$ plant arrangement interactions were evident for both sunburned fruit weight and total cull fruit weight in Texas. The pattern of the two interactions was almost identical, confirming that sunburned fruit weight was an important contributor to the total cull production. Plant arrangement did not affect cull fruit weight in 'X3R Wizard'. Cull fruit weights also did not differ between S30, S37.5, and D30 in 'King Arthur', but the two single-row arrangements produced more cull fruit weight than the D37.5 arrangement (interaction means not presented).

Early harvest, 2003. Cultivar effects were inconsistent between locations (Table 4). 'Boynton Bell' was the leading early producer of U.S. No. 1 fruit in Oklahoma, but not in Texas. There was a greater range in total early marketable fruit weights among cultivars in Texas than in Oklahoma, in part because the average weight per marketable fruit differed among cultivars in Texas but not in Oklahoma (Table 4). As in 2002, single rows resulted in a greater overall total early fruit weight than double rows in Oklahoma, and this was attributed to a greater weight of U.S. No. 1 fruit from single rows in Oklahoma. Although the same overall trend was apparent in Texas, cultivar $\times$ plant arrangement interactions were evident for U.S. No. 1, premium, and total early marketable fruit weights (Table 5). Because premium and total marketable fruit weights incorporated U.S. No. 1 fruit weight, the three interactions were very similar. Within cultivars, single rows gave higher early U.S. No. 1 and premium yields than double rows only for 'Lafayette' in Texas. Within cultivars, row arrangement had no effect on total early marketable fruit weight. Within S30, early marketable yields were higher for 'X3R Wizard' and 'Lafayette' than for the other

Table 3. Full-season bell pepper fruit yields as affected by cultivar and plant arrangement, Uvalde, Tex., 2002. ${ }^{2}$

\begin{tabular}{|c|c|c|c|c|c|c|c|c|c|c|}
\hline Treatment factor & \multicolumn{6}{|c|}{ Marketable fruit wt } & \multicolumn{3}{|c|}{ Cull fruit wt $\left(\mathrm{Mg} \cdot \mathrm{ha}^{-1}\right)$} & $\begin{array}{c}\text { Overall totaly } \\
\text { fruit wt } \\
\left(\mathrm{Mg} \cdot \mathrm{ha}^{-1}\right)\end{array}$ \\
\hline \multicolumn{11}{|l|}{ Cultivar } \\
\hline King Arthur & 1.7 & 20.8 & 22.4 & 10.1 & 32.6 & 108 & 5.0 & 0.06 & 7.8 & 40.4 \\
\hline Significance & NS & $* *$ & $* *$ & NS & $* *$ & NS & $* *$ & NS & $* *$ & $*$ \\
\hline \multicolumn{11}{|l|}{ Plant arrangement ${ }^{t}$} \\
\hline $\mathrm{S} 30$ & 1.7 & 25.9 & 27.6 & 12.3 & 40.0 & 109 & 4.9 & 0.58 & 7.3 & 47.3 \\
\hline $\mathrm{D} 37.5$ & 1.8 & 20.0 & 21.8 & 9.8 & 31.6 & 109 & 2.4 & 0.66 & 4.7 & 36.4 \\
\hline \multicolumn{11}{|c|}{ Contrasts within plant arrangements } \\
\hline Single vs. double rows & NS & ** & ** & * & ** & NS & ** & NS & ** & ** \\
\hline
\end{tabular}

${ }^{2}$ Totals over six harvests from 19 June through 28 Aug. Two of the measured variables showed a significant $(P \leq 0.05)$ cultivar $\times$ plant arrangement interaction: sunburned fruit $(P \leq 0.001)$ and total of all culls $(P \leq 0.001)$.

${ }^{\mathrm{y} O v e r a l l ~ t o t a l}=$ U.S. Fancy + U.S. No. $1+$ U.S. No. $2+$ all culls.

${ }^{x}$ Premium $=$ U.S. Fancy + U.S. No. 1.

${ }^{\mathrm{w} T}$ Total marketable = U.S. Fancy + U.S. No. $1+$ U.S. No. 2.

"Significant $(P \leq 0.05)$ cultivar $\times$ plant arrangement interaction; see text for details.

vAll culls includes misshapen fruit and fruit affected by sunburn, blossom-end rot, or plant pathogens.

tS30 = single rows $0.95 \mathrm{~m}$ apart, plants $30 \mathrm{~cm}$ apart within rows; $\mathrm{D} 30=$ double rows $30 \mathrm{~cm}$ apart on beds, $1.9 \mathrm{~m}$ between centers of beds, plants $30 \mathrm{~cm}$ apart within rows; S37.5 = single rows $0.76 \mathrm{~m}$ apart, plants $37.5 \mathrm{~cm}$ apart within rows; D37.5 = double rows $24 \mathrm{~cm}$ apart on beds, $1.52 \mathrm{~m}$ between centers of beds, plants 37.5 $\mathrm{cm}$ apart within rows.

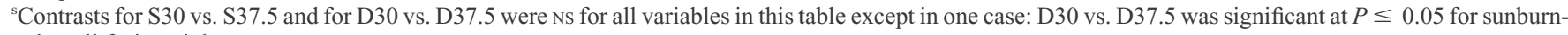
only cull fruit weight.

NS, *, *** Not significant or significant by $F$ test at $P \leq 0.05$ or $\leq 0.01$ respectively. 
Table 4. Bell pepper fruit yields from early harvest as affected by cultivar and plant arrangement, Bixby, Okla. (OK), and Uvalde, Tex. (TX), 2003. ${ }^{\mathrm{z}}$

\begin{tabular}{|c|c|c|c|c|c|c|c|c|c|c|c|c|}
\hline \multirow{2}{*}{ Treatment factor } & \multicolumn{12}{|c|}{ Marketable fruit wt } \\
\hline & \multicolumn{2}{|c|}{ U.S. Fancy $\left(\mathrm{Mg} \cdot \mathrm{ha}^{-1}\right)$} & \multicolumn{2}{|c|}{ U.S. No. $1\left(\mathrm{Mg} \cdot \mathrm{ha}^{-1}\right)$} & \multicolumn{2}{|c|}{$\operatorname{Premium}^{\mathrm{y}}\left(\mathrm{Mg} \cdot \mathrm{ha}^{-1}\right)$} & \multicolumn{2}{|c|}{ U.S. No. $2\left(\mathrm{Mg} \cdot \mathrm{ha}^{-1}\right)$} & \multicolumn{2}{|c|}{$\operatorname{Total}^{\mathrm{x}}\left(\mathrm{Mg} \cdot \mathrm{ha}^{-1}\right)$} & \multicolumn{2}{|c|}{ Avg (g/fruit) } \\
\hline \multicolumn{13}{|l|}{ Cultivar } \\
\hline Karma & 2.1 & 0.6 & $3.4 \mathrm{~b}$ & $5.6 \mathrm{c}$ & $5.5 \mathrm{~b}$ & $6.2 c$ & 0.1 & 1.3 & $5.6 \mathrm{ab}$ & $7.6 \mathrm{c}$ & 194 & $124 \mathrm{a}$ \\
\hline King Arthur & 2.9 & 0.2 & $3.6 \mathrm{~b}$ & $5.2 \mathrm{c}$ & $6.5 \mathrm{ab}$ & $5.4 \mathrm{c}$ & 0.1 & 1.8 & $6.6 \mathrm{ab}$ & $7.3 \mathrm{c}$ & 195 & $100 \mathrm{~b}$ \\
\hline Lafayette & 1.4 & 0.0 & $3.1 \mathrm{~b}$ & $13.6 \mathrm{ab}$ & $4.6 \mathrm{~b}$ & $13.6 \mathrm{ab}$ & 0.1 & 2.8 & $4.6 \mathrm{~b}$ & $16.3 \mathrm{ab}$ & 202 & $126 \mathrm{a}$ \\
\hline X3R Wizard & 1.5 & 0.2 & $2.2 \mathrm{~b}$ & $16.2 \mathrm{a}$ & $3.6 \mathrm{~b}$ & $16.5 \mathrm{a}$ & 0.1 & 1.7 & $3.7 \mathrm{~b}$ & $18.1 \mathrm{a}$ & 196 & $122 \mathrm{a}$ \\
\hline S30 & 2.4 & 0.4 & 4.2 & 11.0 & 6.6 & 11.4 & 0.1 & 1.9 & 6.6 & 13.3 & 193 & 119 \\
\hline D30 & 2.0 & 0.1 & 3.0 & 8.8 & 5.0 & 8.9 & 0.1 & 1.8 & 5.1 & 10.7 & 193 & 116 \\
\hline Significance & NS & NS & $*$ & $*$ & * & $* *$ & NS & NS & $*$ & $*$ & NS & NS \\
\hline
\end{tabular}

${ }^{2}$ In Oklahoma, early harvest occurred on 24 June and 3 July. None of the measured variables showed a significant $(P \leq 0.05)$ cultivar $\times$ plant arrangement interaction. In Texas, early harvest occurred 17 June through 11 July. U.S. No. 1, premium, and total marketable fruit weights all showed significant $(P \leq 0.05)$ cultivar $\times$ plant arrangement interactions. In both locations, there were too few cull fruit for statistical analysis.

${ }^{\mathrm{y}}$ Premium $=$ U.S. Fancy + U.S. No. 1.

${ }^{x}$ Total marketable $=$ U.S. Fancy + U.S. No. $1+$ U.S. No. 2.

w'Mean separation in columns within cultivars by Duncan's multiple range test, $P \leq 0.05$.

${ }^{\vee}$ Significant $(P \leq 0.05)$ cultivar $\times$ plant arrangement interaction; see Table 5 for details.

u'S30 = single rows $0.95 \mathrm{~m}$ apart, plants $30 \mathrm{~cm}$ apart within rows; D30 = double rows $30 \mathrm{~cm}$ apart on beds, $1.9 \mathrm{~m}$ between centers of beds, plants $30 \mathrm{~cm}$ apart within rows.

NS, *, ** Not significant or significant by $F$ test at $P \leq 0.05$ or $\leq 0.01$ respectively.

three cultivars. Total early marketable yields were unaffected by cultivar within D30, but U.S. No. 1 and premium early yields were higher for 'X3R Wizard' than for 'Karma' and 'King Arthur' (Table 5).

Full-season yields, Oklahoma, 2003. The five tested cultivars yielded similarly in Oklahoma, despite differences in average weight per marketable fruit (Table 6). Single rows resulted in higher total marketable fruit weights than double rows and, as with early yields, this was attributed to a greater weight of U.S. No. 1 fruit from single rows. Total cull fruit weight was unaffected by row arrangement; but, as in 2002, a higher weight of sunburned fruit was produced with single rows than with double rows (Table 6). No cultivar $\times$ plant arrangement interactions were evident.

Full-season yields, Texas, 2003. 'Lafayette' and 'X3R Wizard' produced more U.S. No. 1, premium, and total marketable fruit weight than 'Boynton Bell', 'Karma', and 'King Arthur' in Texas (Table 7). 'Lafayette' and 'X3R Wizard' also had the highest average weights per marketable fruit. Single rows resulted in higher total marketable fruit weights than double rows, which could be attributed to a greater weight of U.S. No. 1 fruit from single rows (Table 7), just as in Oklahoma (Table 6). Single rows led to both higher sunburned fruit weights and total cull fruit weights compared with double rows in Texas (Table 7 ). No cultivar $\times$ plant arrangement interactions were evident.

Plant height and dry weight data. Differences were observed among cultivars. However, in both years and at both locations, there were no significant $(P \leq 0.05)$ main effects of plant arrangement, and cultivar $\times$ plant arrangement interactions were not evident. Therefore, these data are not presented.

\section{Discussion}

Cultivars showed different regional adaptation. All five tested cultivars were potentially useful in Oklahoma. 'Boynton Bell' was notable for its earliness in Oklahoma, and it performed well in previous Oklahoma cultivar trials (Kahn et al., 2003). Choice of cultivar was more critical in Texas, where 'X3R Wizard' consistently outperformed 'King Arthur', than in Oklahoma. These

Table 5. Cultivar $\times$ plant arrangement interactions evident for early marketable bell pepper fruit weight, Uvalde, Tex., 2003.

\begin{tabular}{lcccc}
\hline & \multirow{2}{*}{$\begin{array}{c}\text { Plant } \\
\text { arrangement }\end{array}$} & \multicolumn{3}{c}{ Early marketable fruit wt $\left(\mathrm{Mg} \cdot \mathrm{ha}^{-1}\right)^{\mathrm{z}}$} \\
\cline { 2 - 5 } Cultivar & S30 & $18.8 \mathrm{a}$ & Premium & Total \\
\hline X3R Wizard & S30 & $17.2 \mathrm{a}$ & $19.1 \mathrm{a}$ & $21.5 \mathrm{a}$ \\
Lafayette & D30 & $13.7 \mathrm{ab}$ & $17.2 \mathrm{a}$ & $20.1 \mathrm{ab}$ \\
X3R Wizard & D30 & $9.9 \mathrm{bc}$ & $9.9 \mathrm{bc}$ & $14.8 \mathrm{abc}$ \\
Lafayette & S30 & $9.4 \mathrm{bc}$ & $9.4 \mathrm{bc}$ & $12.6 \mathrm{bc}$ \\
Boynton Bell & D30 & $8.5 \mathrm{bc}$ & $8.6 \mathrm{bc}$ & $10.8 \mathrm{c}$ \\
Boynton Bell & D30 & $6.6 \mathrm{c}$ & $6.6 \mathrm{c}$ & $10.5 \mathrm{c}$ \\
Karma & D30 & $5.3 \mathrm{c}$ & $5.4 \mathrm{c}$ & $8.6 \mathrm{c}$ \\
King Arthur & S30 & $5.2 \mathrm{c}$ & $5.5 \mathrm{c}$ & $6.8 \mathrm{c}$ \\
King Arthur & S30 & $4.6 \mathrm{c}$ & $5.9 \mathrm{c}$ & $7.8 \mathrm{c}$ \\
Karma & & & $6.5 \mathrm{c}$ \\
\hline
\end{tabular}

${ }^{\mathrm{z}}$ Values are least square means. Within columns, means followed by the same letter do not differ by $t$-test $P=0.05$. findings reinforce the need for current, localized cultivar recommendations.

Row arrangement did not consistently affect early marketable yields. There was a greater early weight of U.S. No. 1 fruit from single rows than from double rows in Oklahoma in both years, but not in Texas. Also, most of the cultivar $\times$ plant arrangement interactions that were evident in Texas affected early fruit production rather than full-season yield. Main effects of plant arrangement are less pronounced before plants achieve their maximum size, at which time potential competitive effects would increase (Wagenmakers and Callesen, 1995). Wilcox (1970) compared single- and twin-row patterns of tomatoes in the same population and found early ripe fruit harvests were similar.

Single rows resulted in more full-season total marketable fruit weight than double rows in three experiments out of four. Production of U.S. Fancy fruit was not high under conditions of these studies and was unaffected by plant arrangement. Full-season weights of U.S. No. 2 fruit were affected by plant arrangement only in Texas in 2002 (Table 3). Thus, the increase in total marketable fruit weight was primarily the result of an increased weight of U.S. No. 1 fruit with single rows. Wilcox (1970) reported that twin-row patterns of tomatoes led to higher full-season ripe fruit yields than single-row patterns only at the highest of four comparable plant populations. Frost and Kretchman (1988) compared two tomato cultivars at four plant populations in both single and twin rows and found that the twin-row configuration increased yields in only one of the two cultivars. Both the Wilcox (1970) and Frost and Kretchman (1988) studies involved onceover harvests. With a multiple sequential harvest system, as was used in the current studies, Fery and Janick (1970) found that a single-row pattern outyielded a square 
Table 6. Full-season bell pepper fruit yields as affected by cultivar and plant arrangement, Bixby, Okla., 2003. ${ }^{\mathrm{z}}$

\begin{tabular}{|c|c|c|c|c|c|c|c|c|c|}
\hline \multirow[b]{2}{*}{ Treatment factor } & \multicolumn{6}{|c|}{ Marketable fruit wt } & \multicolumn{2}{|c|}{ Cull fruit wt $\left(\mathrm{Mg} \cdot \mathrm{ha}^{-1}\right)$} & \multirow[b]{2}{*}{$\begin{array}{l}\text { Overall total }^{\mathrm{y}} \\
\text { fruit wt }\left(\mathrm{Mg} \cdot \mathrm{ha}^{-1}\right.\end{array}$} \\
\hline & $\begin{array}{l}\text { U.S. Fancy } \\
\left(\mathrm{Mg} \cdot \mathrm{ha}^{-1}\right)\end{array}$ & 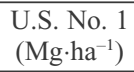 & $\begin{array}{l}\text { Premium }^{\mathrm{x}} \\
\left(\mathrm{Mg} \cdot \mathrm{ha}^{-1}\right)\end{array}$ & $\begin{array}{l}\text { U.S. No. } 2 \\
\left(\mathrm{Mg} \cdot \mathrm{ha}^{-1}\right)\end{array}$ & $\begin{array}{c}\text { Total }^{\mathrm{w}} \\
\left(\mathrm{Mg} \cdot \mathrm{ha}^{-1}\right)\end{array}$ & $\begin{array}{l}\text { Average } \\
\text { (g/fruit) }\end{array}$ & $\begin{array}{l}\text { Sunburn } \\
\text { only }\end{array}$ & $\begin{array}{l}\text { Total of } \\
\text { all culls }\end{array}$ & \\
\hline \multicolumn{10}{|l|}{ Cultivar } \\
\hline Boynton Bell & 3.7 & 18.3 & 22.0 & 5.8 & 27.8 & $148 b^{u}$ & 0.9 & 2.0 & 29.8 \\
\hline Karma & 3.9 & 14.6 & 18.5 & 4.7 & 23.2 & $163 \mathrm{a}$ & 1.0 & 2.3 & 25.5 \\
\hline King Arthur & 4.1 & 16.6 & 20.7 & 5.4 & 26.1 & $167 \mathrm{a}$ & 1.4 & 2.9 & 29.0 \\
\hline Lafayette & 2.0 & 13.1 & 15.0 & 8.6 & 23.7 & $166 \mathrm{a}$ & 0.6 & 1.9 & 25.5 \\
\hline X3R Wizard & 2.3 & 15.8 & 18.1 & 5.9 & 24.0 & $159 \mathrm{ab}$ & 0.5 & 1.7 & 25.6 \\
\hline Significance & NS & NS & NS & NS & NS & $*$ & NS & NS & NS \\
\hline \multicolumn{10}{|l|}{ Plant arrangement ${ }^{t}$} \\
\hline S30 & 3.5 & 16.9 & 20.4 & 6.6 & 27.0 & 160 & 1.2 & 2.5 & 29.4 \\
\hline D30 & 2.9 & 14.4 & 17.4 & 5.6 & 22.9 & 161 & 0.6 & 1.8 & 24.8 \\
\hline Significance & NS & $*$ & $*$ & NS & $*$ & NS & $*$ & NS & $*$ \\
\hline
\end{tabular}

${ }^{z}$ Totals over five harvests from 24 June through 28 July. None of the measured variables showed a significant $(P \leq 0.05)$ cultivar $\times$ plant arrangement interaction. ${ }^{\mathrm{y}}$ Overall total $=$ U.S. Fancy + U.S. No. $1+$ U.S. No. $2+$ all culls.

${ }^{x}$ Premium $=$ U.S. Fancy + U.S. No. 1.

${ }^{\mathrm{w}}$ Total marketable = U.S. Fancy + U.S. No. $1+$ U.S. No. 2.

${ }^{v}$ All culls includes misshapen fruit and fruit affected by sunburn, blossom-end rot, or plant pathogens.

"Mean separation in columns within cultivars by Duncan's multiple range test, $P \leq 0.05$.

${ }^{\mathrm{t}} \mathrm{S} 30=$ single rows $0.95 \mathrm{~m}$ apart, plants $30 \mathrm{~cm}$ apart within rows; $\mathrm{D} 30=$ double rows $30 \mathrm{~cm}$ apart on beds, $1.9 \mathrm{~m}$ between centers of beds, plants $30 \mathrm{~cm}$ apart within rows.

NS, * Not significant or significant by $F$ test at $P \leq 0.05$.

Table 7. Full-season bell pepper fruit yields as affected by cultivar and plant arrangement, Uvalde, Tex., 2003. ${ }^{2}$

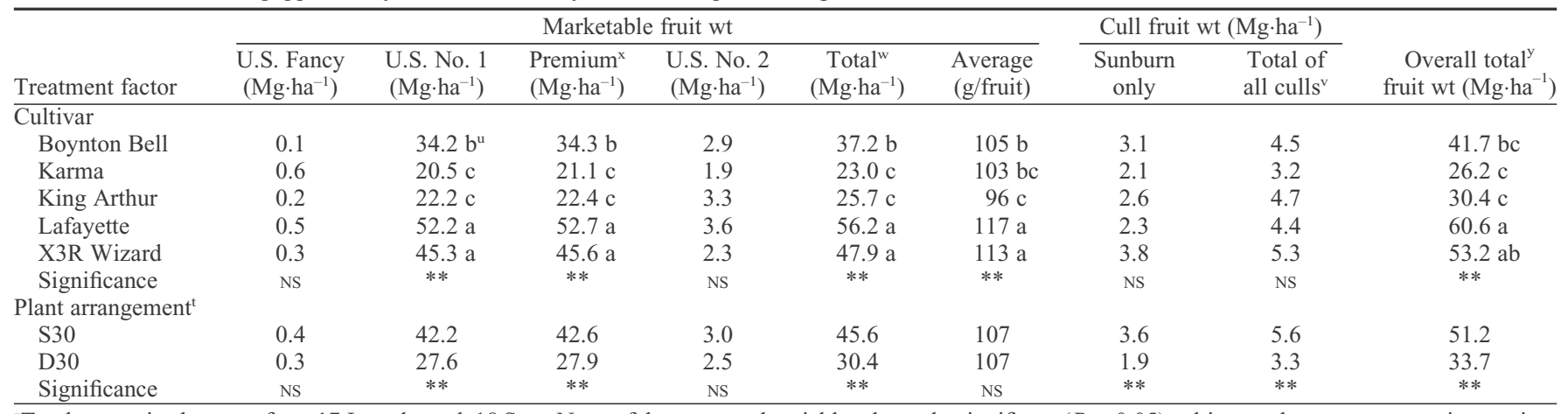

${ }^{2}$ Totals over nine harvests from 17 June through 18 Sept. None of the measured variables showed a significant $(P \leq 0.05)$ cultivar $\times$ plant arrangement interaction. ${ }^{\mathrm{y}}$ Overall total $=$ U.S. Fancy + U.S. No. $1+$ U.S. No. $2+$ all culls.

${ }^{\times}$Premium $=$U.S. Fancy + U.S. No. 1.

${ }^{\mathrm{w} T o t a l}$ marketable = U.S. Fancy + U.S. No. $1+$ U.S. No. 2.

${ }^{v}$ All culls includes misshapen fruit and fruit affected by sunburn, blossom-end rot, or plant pathogens.

"Mean separation in columns within cultivars by Duncan's multiple range test, $P \leq 0.05$.

${ }^{\text {tS } 30}=$ single rows $0.95 \mathrm{~m}$ apart, plants $30 \mathrm{~cm}$ apart within rows; D30 = double rows $30 \mathrm{~cm}$ apart on beds, $1.9 \mathrm{~m}$ between centers of beds, plants $30 \mathrm{~cm}$ apart within rows.

NS, ** Not significant or significant by $F$ test at $P \leq 0.01$.

pattern when averaged across five tomato plant populations per unit area. It appears that, at the population density of one plant every $0.285 \mathrm{~m}^{2}$ used in the current studies, the more square planting patterns achieved with the double rows decreased the net spacing between plants and resulted in more plant-toplant competition, most likely for light. Jaaffar and Gardner (1988) showed that canopy closure and light interception were greater in twin rows [ $(0.69-0.23) \times 0.15 \mathrm{~m}]$ than in conventional single rows $(0.91 \mathrm{~m})$ in peanuts (Arachis hypogaea L.). This proved advantageous to peanut yield at a common plant population density only when the twin rows resulted in greater spacing between plants compared with the single rows.

Average weight per marketable fruit was consistently unaffected by plant arrangement. Others have reported similar results in peppers (Ahmed, 1984; Gaye et al., 1992; Stoffella and Bryan, 1988).

As hypothesized, use of double rows rather than single rows tended to reduce production of cull fruits (Tables 2,3,6 and 7). This occurred primarily through effects on sunburned fruits. Plants in multiple-row arrangements help to shade each other (Locascio and Stall, 1994; Sayre, 1959). Sayre (1959) stated that tomato plants grown in twin rows produced high-quality fruits with almost no blossom-end rot compared with those grown in single rows. Significant effects of plant arrangement on blossom-end rot were seen only in Oklahoma in 2002 in the current studies (Table 2).

Cultivar $\times$ plant arrangement interactions were not evident in Oklahoma and never involved full-season marketable fruit weights at either location in either year.
Locascio and Stall (1994) varied row arrangement, in-row plant spacing, and $\mathrm{N}$ rate on bell peppers, and no significant interactions were evident. There also were no cultivar $\times$ plant arrangement interactions for most yield measurements in two studies in which different peanut cultivars were planted in varying arrangements at a uniform plant population density (Gardner and Auma, 1989; Jaaffar and Gardner, 1988).

Given the tested population of one plant every $0.285 \mathrm{~m}^{2}$, these studies indicate that a single-row arrangement is likely to result in higher full-season U.S. No. 1 bell pepper fruit yields than a double-row arrangement, despite an increased potential for cull fruit production with single rows. The findings should be applicable across a range of cultivars. 


\section{Literature Cited}

Ahmed, M.K. 1984. Optimum plant spacing and nitrogen fertilization of sweet pepper in the Sudan Gezira. Acta Hort. 143:305-310.

Batal, K.M. and D.A. Smittle. 1981. Response of bell pepper to irrigation, nitrogen and plant population. J. Amer. Soc. Hort. Sci. 106:259262.

Fery, R.L. and J. Janick. 1970. Effect of planting pattern and population pressure on the yield response of tomato. HortScience 5: 443-444.

Frost, D.J. and D.W. Kretchman. 1988. Plant spatial arrangement and density effects on small- and medium-vined processing tomatoes. J. Amer. Soc. Hort. Sci. 113:51-55.

Gardner, F.P. and E.O. Auma. 1989. Canopy structure, light interception, and yield and market quality of peanut genotypes as influenced by planting pattern and planting date. Field Crops Res. 20:13-29.

Gaye, M.M., P.A. Jolliffe, and A.R. Maurer. 1992. Row cover and population density effects on yield of bell peppers in south coastal British Columbia. Can. J. Plant Sci. 72:901-909.

Jaaffar, Z. and F.P. Gardner. 1988. Canopy development, yield, and market quality in peanut as affected by genotype and planting pattern. Crop Sci. 28:299-305.

Kahn, B.A., D.I. Leskovar, B. Bostian, and C. Maness. 2003. Bell pepper culture and cultivar update, p. 109-111. In: Proc. $22^{\text {nd }}$ Annu. Hort. Industries Show. Springdale, Ark., 10-11 Jan. 2003.

Locascio, S.J. and W.M. Stall. 1994. Bell pepper yield as influenced by plant spacing and row arrangement. J. Amer. Soc. Hort. Sci. 119 899-902.

Sayre, C.B. 1959. Spacing of cannery tomatoes. Proc. Amer. Soc. Hort. Sci. 73:305-311.

Stoffella, P.J. and H.H. Bryan. 1988. Plant population influences growth and yields of bell pepper. J. Amer. Soc. Hort. Sci. 113:835839

Wagenmakers, P.S. and O. Callesen. 1995. Light distribution in apple orchard systems in relation to production and fruit quality. J. Hort. Sci. 70:935-948.

Wilcox, G.E. 1970. Influence of row spacing and plant density on single harvest tomato yields. J. Amer. Soc. Hort. Sci. 95:435-437.

Willey, R.N. and S.B. Heath. 1969. The quantitative relationship between plant population and crop yield. Adv. Agron. 21:281-321. 